\title{
Organic position sensitive photodetectors based on lateral donor-acceptor concentration gradients
}

\author{
Juan Cabanillas-Gonzalez, ${ }^{1, a)}$ Ovidio Peña-Rodríguez, ${ }^{2,3,4}$ Inma Suarez Lopez, ${ }^{5}$ \\ Malte Schmidt, ${ }^{2}$ M. Isabel Alonso, ${ }^{2}$ Alejandro R. Goñi, ${ }^{2,6}$ and Mariano Campoy-Quiles ${ }^{2}$ \\ ${ }^{1}$ Instituto Madrileño de Estudios Avanzados en Nanociencia (IMDEA-Nanociencia), Cantoblanco, \\ 28049-Madrid, Spain \\ ${ }^{2}$ Institut de Ciència de Materials de Barcelona (ICMAB-CSIC), Esfera UAB, Bellaterra 08193, Spain \\ ${ }^{3}$ Centro de Microanálisis de Materiales, Universidad Autónoma de Madrid (CMAM-UAM), Cantoblanco, \\ E-28049 Madrid, Spain \\ ${ }^{4}$ Instituto de Óptica, Consejo Superior de Investigaciones Científicas (IO-CSIC), C/Serrano 121, \\ E-28006 Madrid, Spain \\ ${ }^{5}$ Dipartimento di Fisica, Politecnico di Milano, Pza. Leonardo da Vinci 32, Milano 20133, Italy \\ ${ }^{6}$ Institució Catalana de Recerca i Estudis Avançats (ICREA), Passeig Lluis Companys 23, 08010 Barcelona, \\ Spain
}

(Received 7 June 2011; accepted 7 August 2011; published online 8 September 2011)

\begin{abstract}
We report on an organic photodiode configuration based on a donor/acceptor bilayer with opposed complementary thickness gradients. We rely on the strong dependence of the optical field on thickness to achieve spatially dependent spectral responses, a proof-of-concept for position sensitive detection. Ratiometric photocurrent values at two wavelengths allow for position determination independently of the light intensity with a spatial resolution below $600 \mu \mathrm{m}$. (C) 2011 American Institute of Physics. [doi:10.1063/1.3631731]
\end{abstract}

The rich variety of organic compounds with absorption spanning from the UV to the near-IR offers unique possibilities for light harvesting and spectral selectivity in photodetectors. Appropriate combination of compounds with donor/ acceptor character aims at improving charge generation, simultaneously extending the spectral sensitivity range. ${ }^{1,2}$ When donors and acceptors are processed on a multilayer configuration, a strong dependence of the spectral response with the layers thickness is found. This is mainly attributed to two facts. First of all, the incoming and reflected wavefronts at the layer interfaces create a thickness dependent interference pattern of the optical field which determines the light intensity distribution across the device active area. ${ }^{3,4}$ In addition, changes in layer thickness will shift the light harvesting zone in depth with a subsequent effect on charge collection efficiency (the so-called inner filter effect). In general, control of layer thicknesses is crucial for optimization of photodiode efficiencies, whereas in-plane thickness inhomogeneity is often regarded as an unwilling effect. ${ }^{5}$ Nevertheless, a gradual change in layer thickness along the device active layer implemented in a controlled manner enables spectral response tunability, a concept with interesting implications in the field of light detection.

In this work, we have developed donor-acceptor bilayers with opposed lateral thickness gradients along the longitudinal direction. Photodiodes based on this configuration exhibit a variable spectral response parallel to the gradient direction, which constitutes an innovative concept for a position sensitive detector (PSD). This type of photodetector offers potential advantages compared to conventional PSDs based on lateral photoelectric effect. These latter devices monitor the position of light impinging on the active area through the

\footnotetext{
${ }^{\text {a) }}$ Author to whom correspondence should be addressed. Electronic mail: juan.cabanillas@imdea.org.
}

change in lateral photocurrent at the interface between a highly conductive and a resistive semiconducting layer., The proposed approach, in contrast, consists of monitoring light position relying directly on lateral gradients and changes in photocurrent across the active layer (typically few hundred nanometers). Thus, the dynamic range of the photodetector proposed here is not limited by the moderate lateral conductivity of resistive layers. The development of large lateral gradients will enable position monitoring over long displacements without compromising spatial resolution. Reassuringly, this is achieved in a single pixel through a gradual change of the active layer, thus retaining the simplicity of continuous PSDs compared to discrete or pixelated devices.

Photodiodes consisted of copper pthalocyanine $(\mathrm{CuPc})$ and fullerene (C60) bilayers evaporated on two separated stages on top of an indium tin oxide (ITO) anode. Gradual variation in layer thickness was achieved by using a mechanically controlled shutter with gradual displacement along the longitudinal direction. Opposed thickness variation of C60 layer was obtained by rotating the substrate $180^{\circ}$ on the plane prior to deposition and using same evaporation parameters as for $\mathrm{CuPc}$. Aluminum cathodes were deposited on top of $\mathrm{C} 60$ in a third evaporation process. The photocurrent rig consisted of a tungsten halogen lamp coupled to a monochromator with $1 / 8 \mathrm{~m}$ focal length and 600 lines/mm grating. Light impinging on the photodiode was mechanically chopped being the photocurrent response demodulated by a lock-in amplifier. Photocurrent action spectra were obtained upon normalization of the photocurrent spectra by the response provided by a calibrated Si photodiode. Raman characterization was carried out with a LabRam HR800 spectrometer (Jovin Yvon) with the $514 \mathrm{~nm} \mathrm{Ar}+$ excitation line coupled through an Olympus microscope into an argon filled sample chamber. The laser spot was less than $5 \mu \mathrm{m}$ in diameter. 

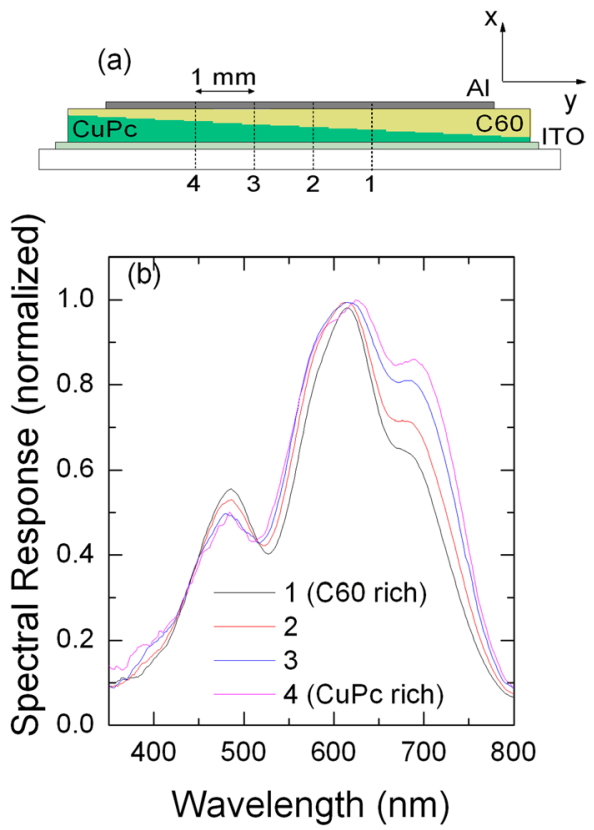

FIG. 1. (Color online) (a) Device configuration showing a thickness gradient of $\mathrm{CuPc} / \mathrm{C} 60$ layers along the longitudinal direction (y-axis). (b) Normalized spectral response at four different positions along the longitudinal direction. Positions (1)-(4) are separated by $1 \mathrm{~mm}$ going from a C60-rich to a CuPc-rich area.

Figure 1(a) displays the concept: a spatially dependent donor/acceptor layer thickness leading to a variable in-plane spectral response. The CuPc layer has a variable thickness comprising $10 \mathrm{~nm}-100 \mathrm{~nm}$ whereas the $\mathrm{C} 60$ layer has a complementary thickness gradient in the same direction such that the total thickness of the bilayer is approximately constant. Light impinging in the active area produces a spectral response which depends on the relative $\mathrm{CuPc} / \mathrm{C} 60$ thicknesses on that position. An example of this behavior is depicted in Figure 1(b). A gradual enhancement of the spectral response in the near-IR is observed by moving the illumination spot from the $\mathrm{C} 60$-rich region towards the $\mathrm{CuPc}$ rich region in $1 \mathrm{~mm}$ steps.

In order to rationalize this behavior, we have monitored the lateral gradient composition present in the device active area using Raman spectroscopy. CuPc and C60 exhibit specific Raman modes which allow for the determination of relative lateral compositions. Dominant Raman active peaks are centered at $1342 \mathrm{~cm}^{-1}(\mathrm{P} 1), 1452 \mathrm{~cm}^{-1}(\mathrm{P} 2)$, and 1528 $\mathrm{cm}^{-1}$ (P4) in CuPc and $1467 \mathrm{~cm}^{-1}$ (P3) in C60 (Figure 2(a)). Figure 2(b) depicts the $\mathrm{P} 4 / \mathrm{P} 3$ intensity ratio as the probe moves along the longitudinal direction in $100 \mu \mathrm{m}$ steps. The enhancement of P4 with respect to P3 confirms an enriched $\mathrm{CuPc}$ concentration as we move along the gradient. The peak ratio profile follows a polynomial dependence instead of linear, as one would initially expect from the constant displacement of the shutter during the evaporation process. This deviation may be related to the cone of evaporation yielding different deposition rates as the uncovered region is located far off the center axis.

The dependence of photocurrent on longitudinal position (y) was predicted by taking into account the thickness gradient determined by the Raman profile and the distribu-
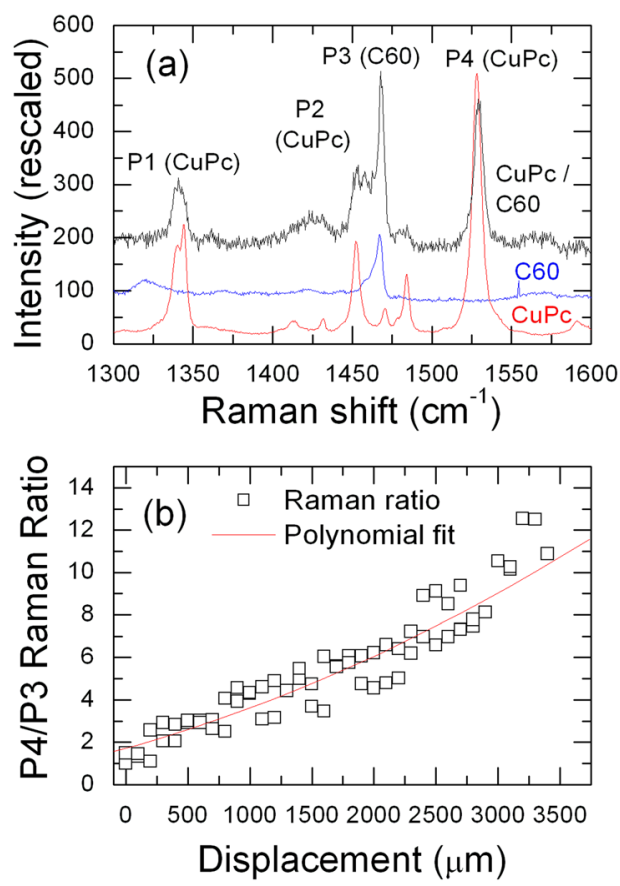

FIG. 2. (Color online) (a) Rescaled Raman spectra of $\mathrm{CuPc}, \mathrm{C} 60$ and $\mathrm{CuPc} /$ C60 bilayer. (b) Raman peak intensity ratio (P4/P3) as a function of displacement in the longitudinal direction (along the gradient) moving from the C60-rich region to the CuPc-rich region. The experimental data was fitted using a quadratic polynomial law (solid line).

tion of the optical field intensity $\left(|E(x, y, \lambda)|^{2}\right)$ modeled by means of matrix methods ${ }^{4}$. This methodology takes advantage of the fact that stratified structures with isotropic and homogeneous media and parallel-plane interfaces can be described by $2 \times 2$ matrices, because the equations governing the propagation of the electromagnetic field are linear and the tangential component of the electromagnetic field is continuous. ${ }^{89}$ Although the assumption of parallel planes might be questionable in graded structures, it should be noted that the thickness variation of each separate layer is done in steps larger than the wavelength of light (see Fig. 1). The good fits obtained confirm the validity of this calculation method. The optical field distribution stems from the superposition of the incident and back reflected light at the different interfaces, with an important contribution arising from the C60/Al interface. Since different thicknesses lead to different interference conditions, marked changes in optical field distribution with layer thickness are expected. Besides layer thickness, the complex refractive index for each layer is used as an input in the modeling. They were deduced from spectroscopic ellipsometric data ${ }^{9}$ (not shown). The photocurrent, $J$, at a given position is proportional to the light absorbed within a region close to the CuPc-C60 boundary delimited between $L(y)$ and $L(y)-2 d$. $L(y)$ and $d$ stand for the $\mathrm{CuPc}$ layer thickness at each $y$ position and the CuPc exciton diffusion length (around $15 \mathrm{~nm}$ (Ref. 10)), respectively. As a first approximation, we may assume that all excitons created between $L(y)-2 d$ and $L(y)$ dissociate with the same probability. Thus $J$ can be estimated as

$$
J(y, \lambda) \propto \int_{L(y)-2 d}^{L(y)} \alpha(\lambda)|E(x, y, \lambda)|^{2} d x
$$




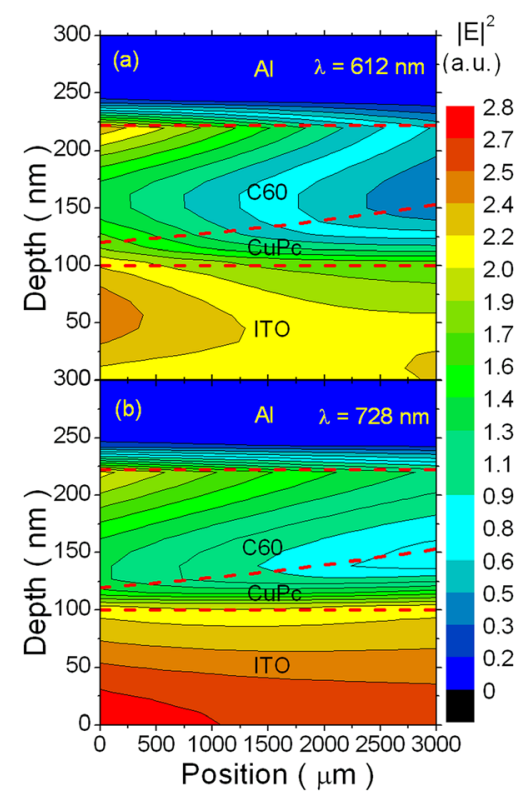

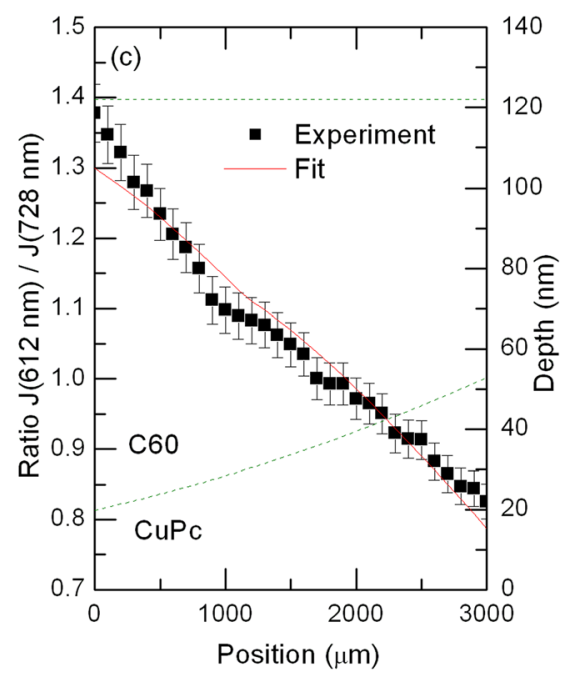

FIG. 3. (Color online) In-depth distribution of the optical field as a function of longitudinal position for (a) $\lambda=612 \mathrm{~nm}$ and (b) $\lambda=728 \mathrm{~nm}$. (c) Photocurrent ratio at $612 \mathrm{~nm}$ and $728 \mathrm{~nm}$ (squares) as a function of position. The spatial dependence of the photocurrent was fitted (solid line) according to the field distributions at $612 \mathrm{~nm}$ and $728 \mathrm{~nm}$. Dashed lines indicate the thickness profiles of $\mathrm{CuPc}$ and $\mathrm{C} 60$ layers estimated by the fit. where $\alpha(\lambda)$ is the absorption coefficient. Figures 3(a) and 3 (b) depict the dependence of $|E|^{2}$ with depth and longitudinal position for the wavelengths $612 \mathrm{~nm}$ (a) and $728 \mathrm{~nm}$ (b). In both cases, the field at the CuPc-C60 boundary is larger for $\mathrm{C} 60$-rich positions. As we move towards the $\mathrm{CuPc}$-rich region, the separation between $\mathrm{Al}$ electrode and the $\mathrm{CuPc}$ C60 boundary decreases, thus modifying the interference pattern within the active layer. This results in a change in $|E|^{2}$ and consequently in photocurrent along the longitudinal direction. In an ideal situation where the intensity of the incoming light is known, the value of the photocurrent would be correlated to the position where light is impinging on the device and this would constitute a basic PSD operation. In practice, it is desirable to self-normalize the signal in order to account for light intensity fluctuations. This mode of operation can be provided by measuring the photocurrent ratio $(R)$ at two wavelengths.

For practical reasons, $R$ values were measured by averaging photocurrents at fixed wavelengths during $5 \mathrm{~s}$ intervals in a sequential manner. Extension of this principle to applications involving large operation bandwidth requires schemes combining broadband illumination with simultaneous detection and discrimination of wavelength dependent signal. Figure 3(c) displays the photocurrent ratio measured at two wavelengths, $628 \mathrm{~nm}$ and $712 \mathrm{~nm}$, from position 1 to position 4 in $100 \mu \mathrm{m}$ intervals. Taking into account the experimental error bars, we estimate a position sensitivity of ca. $600 \mu \mathrm{m}$ over a range of $4 \mathrm{~mm}$. It is noteworthy that, in this work, we restrict the characterization to a length range of $4 \mathrm{~mm}$ within the pixel, although its total length was 1.5 $\mathrm{cm}$. Dynamic ranges extending up to several centimeters can be in principle achieved without additional technical issues. Comparison between experimental values and those predicted from the $|E|^{2}$ distribution yield a good agreement except for a deviation observed at low $L$ values. The reason for this deviation could be related to the assumption that excitons harvested within the region comprising $L-2 d$ and $L$ dissociate exclusively at the CuPc-C60 boundary. At low $L$ values, i.e. $L<2 d$, this assumption fails since exciton dissociation at the ITO/CuPc interface competes with dissociation at the donor-acceptor boundary. This could explain the enhancement in the photocurrent ratio with respect to the prediction based purely on charge photogeneration at the donor-acceptor boundary layer.

In conclusion, we demonstrated that photodiodes based on donor-acceptor bilayers with a longitudinal distribution of thicknesses display an effective variation in photocurrent response along the same direction, which yields from the optical field distribution inside the bilayer. This concept can be applied for position detection achieving a resolution of 600 $\mu \mathrm{m}$ in not fully optimized structures. Since the photocurrent is governed by the electromagnetic field around the interface between the two organic layers, further optimization is envisaged by fine tuning the layer thicknesses, and the use of optical spacers. This strategy can be readily adopted to develop large area position sensitive detectors on flexible substrates with targeted color response.

J.C-G and M.C-Q acknowledge funding from the Spanish Ministry of Science and Innovation through the Ramon y Cajal program, as well as through projects TEC2010-21830C02-02, MAT2009-10642 and PLE2009-0086. Raman measurements were performed at MATGAS 2000 AIE.

\footnotetext{
${ }^{1}$ A. Hadipour, B. de Boer, and P. W. M. Blom, Adv. Funct. Mater. 18, 169 (2008).

${ }^{2}$ M. T. Lloyd, J. E. Anthony, and G. G. Malliaras, Mater. Today 10, 34 (2007).

${ }^{3}$ T. Stübinger and W. Brütting, J. Appl. Phys. 90, 3632 (2001).

${ }^{4}$ L. A. A. Pettersson, L. S. Roman, and O. Inganäs, J. Appl. Phys. 86, 487 (1999).

${ }^{5}$ A. C. Mayer, S. R. Scully, B. E. Hardin, M. W. Rowell, and M. D. McGehee, Mater. Today 10, 28 (2007).

${ }^{6}$ B. P. Rand, J. Xue, M. Lange, and S. R. Forrest, IEEE Photonics Technol. Lett., 15, 1279 (2003).

${ }^{7}$ D. Kabra, Th. B. Singh, and K. S. Narayan, Appl. Phys. Lett. 85, 7073 (2004).

${ }^{8}$ Z. Knittl, Optics of Thin Films (John Wiley \& Sons, London, 1976).

${ }^{9}$ R. M. A. Azzam and N. M. Bashara, Ellipsometry and Polarized Light (North-Holland, Amsterdam, 1977).

${ }^{10}$ P. Peumans, A. Yakimov, and S. R. Forrest, J. Appl. Phys. 93, 3693
} (2003). 